\title{
Vibration Suppression in Smart Thin Beams with Piezoelectric Actuators under a moving Load/Mass Accounting for Large Deflections of the Base Structure
}

\author{
Ali Nikkhoo ${ }^{*}$, Mahnaz Amankhani ${ }^{2}$ and Hamed Ghafari ${ }^{1}$ \\ 'Department of Civil Engineering, Islamic Azad University, Chalous Branch, \\ Chalous, Mazandaran, Iran; nikkhoo@iauc.ac.ir, ghafari@iauc.ac.ir \\ 2Department of Civil Engineering, Islamic Azad University, South Tehran Branch, \\ Tehran, Iran; amankhani@yahoo.com
}

\begin{abstract}
In this article, the motion equations of a single span Euler-Bernoulli beam with geometrically nonlinear behavior under an arbitrary dynamic loading are derived via the Hamilton's Principle. In order to actively control the response of the structure, piezoceramic patches bonded on the lower surface of the beam are utilized. Employing the Eigen Function Expansion Method and considering the first vibrational mode, an equivalent linear control algorithm based on the well-known classical linear optimal control algorithm with displacement-velocity feedback is proposed. Numerical examples for a simply supported beam with immovable-immovable and immovable-movable axial boundary conditions are presented under a moving load and mass excitations. By using a single piezoceramic patch bonded symmetrically at the beam mid-span, the deflection of the beam is decreased into any required levels for both linear and nonlinear behavior of the base beam and therefore, the good performance of the proposed control algorithm is proved.
\end{abstract}

Keywords: Active Control, Eigenfunction Expansion Method, Large Deflections, Moving Mass, Piezoelectric Actuators, Thin Beams

\section{Introduction}

Dynamic effects of moving vehicles traversing the bridge structures has been one the major concerns of structural engineers for more than a century. These structures could be modeled and analyzed by a beam or a plate structure under a moving system. Fryba ${ }^{1}$ presented a valuable study on miscellaneous structures excited by a moving load/ mass/oscillator or system. In most of the cases, except for the moving mass excitation, the closed-form solution was obtained via Laplace-Carson transformation and different aspects of the problem were scrutinized.
However, in the moving mass problems, according to the induced complexity in the formulation, numerical or semi-analytical methods should be exploited. In this regard, many papers have been published dealing with beams ${ }^{2-12}$ and plates ${ }^{13-17}$ traversed by the moving mass. In all of these studies, it has been emphasized that the inertial effects of moving force could not be denied, especially, for high values of moving mass weights and velocities.

On the other hand, vibration suppression of structural systems under environmental excitations has received extensive attention by engineers and researchers during the last decades. One of the efficient methods in this way

${ }^{*}$ Author for correspondence 
is the active structural control. Soong ${ }^{18}$ has introduced a wide range of active control algorithms and systems which could be used in civil engineering structures. Concerning the active control devices and mechanisms, by the emerge of new materials with distinguished properties, called as smart materials, the application of active structural control received more attentions by the scientific communities. Piezoelectric materials are one of those smart materials with the ability of being used both as sensor and actuator. Song et al. ${ }^{19}$ reviewed various applications of piezoceramics in vibration suppression of civil engineering structures used as stacks or patches. Sung ${ }^{20}$ utilized the piezoelectric patches as actuators to decrease the response of a simply supported thin beam under a moving mass. Rofooei and Nikkhoo ${ }^{21}$ derived the governing motion equation of a Kirchhoff plate with a number of bonded piezoelectric patches on its lower surface which was excited by a moving mass traveling on an arbitrary trajectory. They employed the classical linear optimal control algorithm with displacement-velocity feedback to reduce the plate's mid-point deflection for any sever type of loading. Recently, Nikkhoo ${ }^{22}$ scrutinized the capability of the classical linear optimal control algorithm with displacement-velocity and velocity-acceleration feedbacks in vibration suppression of single and multi-span thin beams with piezoelectric actuators and small deformations of the base structure under impulsive loading as well as the moving mass one. He proved the efficiency and high performance of the employed control algorithm for different types of loading.

In contrast to the above-mentioned, fewer studies have been devoted to the beam or plate structures under dynamic loads considering the geometrically nonlinear behavior of the base structure. Hino et al. ${ }^{23}$ explored a simply supported beam under a moving load and nonlinear behavior of the beam via finite element method. Yoshimura et al. ${ }^{24}$ used Galerkin method to solve the similar problem. Their results revealed because of the geometric stiffness which is added to the structural system, the nonlinear response is smaller than the linear one for different load speeds. Kiani and Nikkhoo ${ }^{25}$ investigated the behavior of thin beams with large deflections and different boundary conditions traversed by a moving mass via the generalized Kernel Particle Method (RKPM). They determined the discrepancies between the results obtained by the linear and nonlinear behavior of the beam for different values of the moving mass weights and velocities.

In this paper, the constitutive equations of motion of a thin beam with large deflections and a number of piezoelectric patches bonded on its lower surface under an arbitrary external excitation are derived by employing the Hamilton's Principle. By assuming the moving load and moving mass as the excitations, an equivalent linear control algorithm would be used to reduce the mid-point deflection of the beam. The obtained results via eigen function expansion method prove the proposed algorithm to be efficacious.

\section{Problem Formulation}

A single span continuous uniform undamped EulerBernoulli beam with any boundary conditions is assumed and the external excitation is defined as $f(r$, $t)$. The subscripts (b) and (p) are used to deal with the parameters of the beam and the piezoelectric actuators, respectively. The beam flexural rigidity which are assumed to be constant are denoted by $\left(E_{b} I_{b}\right)$ and $(m)$, correspondingly. $w(r, t)$ and $u(r, t)$ are pertinent to the transverse and axial deflections of the beam, respectively for any spatial location, $r$, and time, $t$. The origin of the beam is coincident at its left hand side (Figure 1). The initial conditions are supposed to be $w(x, 0)=g_{1}(x)$, $\partial w(x, 0) / \partial x=g_{2}(x), u(x, 0)=g_{3}(x)$ and $\partial u(x, 0) / \partial x=g_{4}(x)$, where, $g_{1}(x), g_{2}(x), g_{3}(x)$ and $g_{4}(x)$ are any continuous functions. $n$ piezoelectric patches for the actuating purpose are bonded on the lower surface of the beam in which their node locations are identified by $x_{2 i-1}$ and $x_{2 i}$

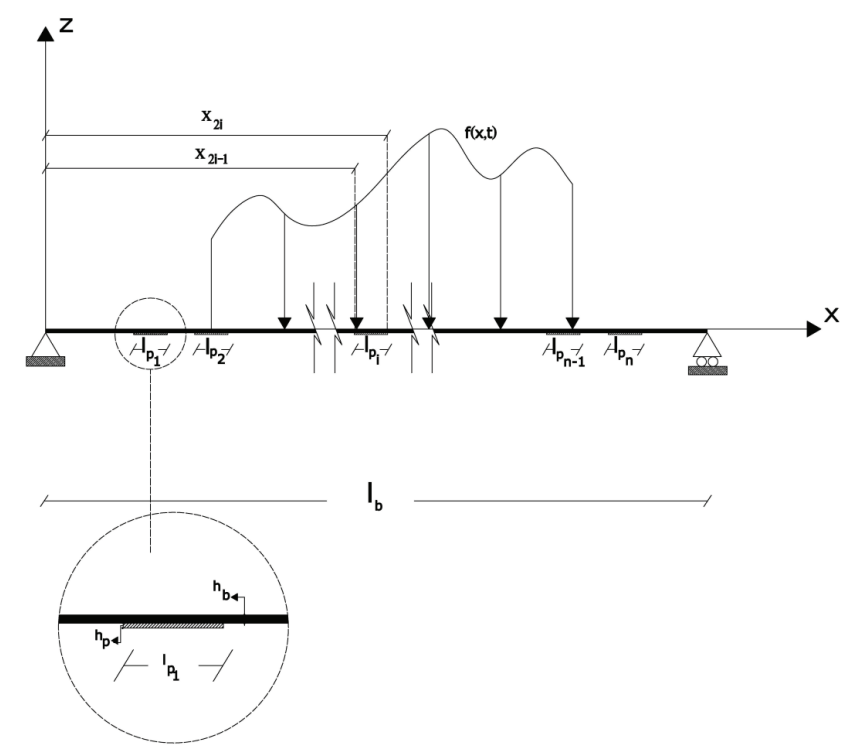

Figure 1. Geometry of a single span smart beam under an arbitrary external excitation with piezoelectric patches bonded on its lower side. 
where, $\mathrm{i}=1,2, \ldots, \mathrm{n}$. According to Figure $1, x_{2 i}-x_{2 i-1}=\left(l_{p}\right)_{i}$ , where, $\left(l_{p}\right)_{i}$, is the length of the $i^{t h}$ piezoelectric patch. The location of the neutral axis of the base beam is supposed to be unchanged according to the size and material properties of the patches in comparison with the host beam. Based on the constitutive equations of piezoelectric materials used as a one-dimensional patch is as follows ${ }^{26,27}$ :

$$
\left[\begin{array}{c}
\sigma_{11} \\
E_{3}
\end{array}\right]=\left[\begin{array}{cc}
E_{p}^{E} & -h_{31} \\
-h_{31} & \beta_{33}^{T}
\end{array}\right]\left[\begin{array}{l}
\varepsilon_{11} \\
D_{3}
\end{array}\right] .
$$

The kinetic energy of the beam-piezo systems accounting for large deflections of the beam is:

$$
T=T_{b}+T_{p}
$$

in which,

$$
T_{b}=\frac{1}{2} \int_{0}^{l_{b}} \rho_{b} A_{b}\left[\frac{\partial^{2} w}{\partial t^{2}}+\frac{\partial^{2} u}{\partial t^{2}}\right] d x,
$$

and

$$
T_{p}=\frac{1}{2} \sum_{i=1}^{n} \int_{0}^{l_{b}} \rho_{p} A_{p}\left[\frac{\partial^{2} w}{\partial t^{2}}+\frac{\partial^{2} u}{\partial t^{2}}\right] \Delta H_{i}(x) d x .
$$

The strain energy of the system can be denoted by:

$$
U=U_{b}+U_{p},
$$

where,

$$
\begin{aligned}
U_{b} & =\frac{1}{2} \int_{V} \varepsilon^{T} \sigma d V=\frac{1}{2} \int_{V} E_{b} \varepsilon_{11}^{2} d V \\
& =\frac{1}{2} \int_{V} E_{b}\left(u_{x}^{2}+\frac{1}{4} w_{x}^{4}+y^{2} w_{x x}^{2}+u_{x} w_{x}^{2}-2 y u_{x} w_{x x}-\frac{1}{2} y w_{x}^{2} w_{x x}\right) d V, \\
& =\frac{E_{b} A_{b}}{2}\left[\int_{0}^{l_{b}}\left(u_{x}^{2}+\frac{1}{4} w_{x}^{4}+u_{x} w_{x}^{2}\right) d x\right]+\frac{E_{b} I_{b}}{2}\left[\int_{0}^{b_{b}}\left(w_{x x}^{2}\right) d x\right]
\end{aligned}
$$

and,

$$
\begin{aligned}
U_{b} & =\frac{1}{2} \int_{V} \varepsilon^{T} \sigma d V=\frac{1}{2} \int_{V} E_{b} \varepsilon_{11}^{2} d V \\
& =\frac{1}{2} \int_{V} E_{b}\left(u_{x}^{2}+\frac{1}{4} w_{x}^{4}+y^{2} w_{x x}^{2}+u_{x} w_{x}^{2}-2 y u_{x} w_{x x}-\frac{1}{2} y w_{x}^{2} w_{x x}\right) d V . \\
& =\frac{E_{b} A_{b}}{2}\left[\int_{0}^{l_{b}}\left(u_{x}^{2}+\frac{1}{4} w_{x}^{4}+u_{x} w_{x}^{2}\right) d x\right]+\frac{E_{b} I_{b}}{2}\left[\int_{0}^{l_{b}}\left(w_{x x}^{2}\right) d x\right]
\end{aligned}
$$

In the above equations, $\rho, A, b, h$ and $l$ signifies the density, cross-section area, width, thickness and length of the beam, correspondingly and:

$$
I_{p}=\frac{1}{12} b_{p} h_{p}^{3}+\frac{1}{4} A_{p}\left(h_{b}+h_{p}\right)^{2}, \quad A_{p}=b_{p} h_{p} .
$$

Besides, $H$ is the Heaviside Step Function and:

$$
\Delta H_{i}(x)=H\left(x-x_{2 i-1}\right)-H\left(x-x_{2 i}\right), \quad i=1,2, \ldots, n .
$$

For the sake of simplicity, the term $\frac{1}{2} u_{x}^{2}$, is neglected in calculation of the strains related to large deflections ${ }^{28}$, i.e.,

$$
\varepsilon_{x}=u_{x}+\frac{1}{2} w_{x}^{2}-y w_{x x} .
$$

in which, $y$ is the distance of any point of the beam section from the neutral line. Moreover, it is assumed there is at least one axis of symmetry in the beam section to achieve Equations 6 and 7. Alternatively, the virtual work done by the external excitation is:

$$
\delta W=\int_{0}^{l_{b}} f(x, t) \delta w d x
$$

The governing motion equations as well as the natural and geometric boundary conditions could be found by applying the Hamilton's Principle.

$$
\delta \mathrm{H}=\delta \int_{t_{1}}^{t_{2}}(T-U+W) d t=0,
$$

By substituting Eqs. 3, 4, 6, 7 and 11 into Eq. 12, one could obtain:

$$
\left\{\begin{array}{l}
\rho_{b} A_{b} \frac{\partial^{2} w}{\partial t^{2}}+E_{b} I_{b} \frac{\partial^{4} w}{\partial x^{4}}+\sum_{i=1}^{n}\left[\rho_{p} A_{p} \frac{\partial^{2} w}{\partial t^{2}}+E_{p}^{E} I_{p} \frac{\partial^{4} w}{\partial x^{4}}\right] \Delta H_{i}(x) \\
=f(x, t)+E_{b} A_{b}\left(\frac{3}{2} w_{x}^{2} w_{x x}+u_{x x} w_{x}+u_{x} w_{x x}\right) \\
-\frac{1}{2} \sum_{i=1}^{n}\left[h_{31} D_{3} A_{p}\left(w_{x x} \Delta H_{i}(x)+w_{x} \Delta \delta_{i}(x)-\left(h_{b}+h_{p}\right) \Delta \delta_{i}^{\prime}(x)\right)\right] \\
\rho_{b} A_{b} \frac{\partial^{2} u}{\partial t^{2}}-E_{b} A_{b} \frac{\partial^{2} u}{\partial x^{2}}+\sum_{i=1}^{n}\left[\rho_{p} A_{p} \frac{\partial^{2} u}{\partial t^{2}}-E_{p}^{E} A_{p} \frac{\partial^{2} u}{\partial x^{2}}\right] \Delta H_{i}(x) \\
=E_{b} A_{b}\left(w_{x} w_{x x}\right)+\sum_{i=1}^{n} h_{31} D_{3} A_{p} \Delta \delta_{i}(x)
\end{array}\right.
$$

in which,

$$
\begin{aligned}
& \Delta \delta_{i}(x)=\delta\left(x-x_{2 i-1}\right)-\delta\left(x-x_{2 i}\right), \\
& \Delta \delta_{i}^{\prime}(x)=\delta^{\prime}\left(x-x_{2 i-1}\right)-\delta^{\prime}\left(x-x_{2 i}\right), \quad i=1,2, \ldots, n .
\end{aligned}
$$

$\delta(x)$ and $\delta^{\prime}(x)$ are the Dirac-Delta function and its first spatial derivative, respectively. In Eq. 13 , the term, $h_{31} D_{3}$, could be replaced by $-\frac{d_{31} E_{p}^{E}}{h_{p}} V(t)$ according to Crawely and de Luis ${ }^{29}$, where, $d_{31}$, is the piezoelectric module and $V(t)$ denotes the applied voltage to the piezoelectric patches. Disregarding the terms pertinent to the piezoelectric patches in Eq. 13, the following equation for an uncontrolled beam with large deflections would be obtained: 


$$
\left\{\begin{aligned}
\rho_{b} A_{b} \frac{\partial^{2} w}{\partial t^{2}}+E_{b} I_{b} \frac{\partial^{4} w}{\partial x^{4}}= & f(x, t)+E_{b} A_{b} \\
& \left(\frac{3}{2} w_{x}^{2} w_{x x}+u_{x x} w_{x}+u_{x} w_{x x}\right) . \\
\rho_{b} A_{b} \frac{\partial^{2} u}{\partial t^{2}}-E_{b} A_{b} \frac{\partial^{2} u}{\partial x^{2}}= & E_{b} A_{b}\left(w_{x} w_{x x}\right)
\end{aligned}\right.
$$

The above coupled equations are in complete agreement by the equations presented by Nayfeh and Mook $^{28}$.

\section{Solution of the Motion Equations}

To solve the motion equations of the uncontrolled beam, the Eigen function expansion method is utilized by assuming the first $n_{1}$ transverse and longitudinal vibration modes as:

$$
\left\{\begin{array}{l}
w(x, t)=\sum_{i=1}^{n_{1}} \phi_{i}(x) A_{i}(t), \\
u(x, t)=\sum_{i=1}^{n_{1}} \psi_{i}(x) B_{i}(t)
\end{array}\right.
$$

in which, $\phi_{i}(x)$ and $\psi_{i}(x)$ are the transverse and longitudinal vibration mode shapes, correspondingly, and $A_{i}(t)$ and $B_{i}(t)$ denotes the time dependent vibration amplitudes. By appropriate substitution of Eq. 16 in Eq. 15:

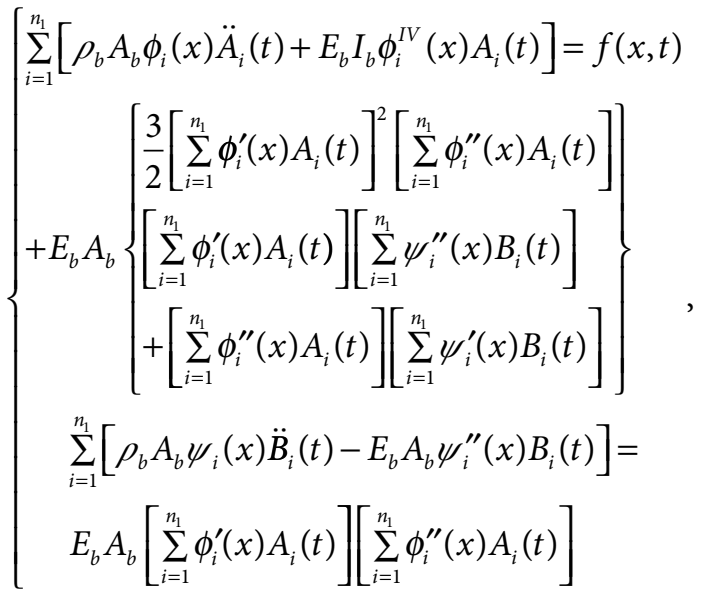

On the other hand, the free vibration of beams for small deformations is:

$$
\left\{\begin{array}{l}
w(x, t)=\sum_{i=1}^{\infty} \phi_{i}(x) e^{j \omega_{i} t} \\
u(x, t)=\sum_{i=1}^{\infty} \psi_{i}(x) e^{j \bar{j}_{i} t}
\end{array}\right.
$$

where, $\omega_{i}$ and $\bar{\omega}_{i}$ are the transverse and longitudinal angular frequencies of the beam, respectively and $j=\sqrt{-1}$ . Replacing Eq. 18 on the left hand side of Eq. 15 leads to:

$$
\left\{\begin{array}{c}
E_{b} I_{b} \phi_{i}^{I V}(x)=\rho_{b} A_{b} \omega_{i}^{2} \phi_{i}(x) \\
E_{b} A_{b} \psi_{i}^{\prime \prime}(x)=-\rho_{b} A_{b} \bar{\omega}_{i}^{2} \psi_{i}(x)
\end{array}\right.
$$

Multiplying both sides of Eq. 17 by $\phi_{j}(x)$ and $\psi_{j}(x)$ respectively, and integrating on the beam length the ordinary differential equations of motions would attain by the use of orthogonality of vibration modes, i.e., $\int_{0}^{l_{b}} \rho_{b} A_{b} \phi_{i}(x) \phi_{j}(x) d x=\delta_{i j}$ and $\int_{0}^{l_{b}} \rho_{b} A_{b} \psi_{i}(x) \psi_{j}(x) d x=\delta_{i j}$ for $i, j=1,2, \ldots, n_{1}$. For the sake of simplicity, only the first mode of the system is considered. Therefore,

$$
\left\{\begin{array}{c}
\ddot{A}(t)+\omega_{1}^{2} A(t)-E_{b} A_{b}\left(\frac{3}{2} \alpha_{1} A^{3}(t)+\alpha_{2} A(t) B(t)\right)=f(t) \\
\ddot{B}(t)+\bar{\omega}_{1}^{2} B(t)-E_{b} A_{b} A^{2}(t)=0
\end{array}\right.
$$

in which,

$$
\left\{\begin{array}{l}
\alpha_{1}=\int_{0}^{l_{b}} \phi_{1}^{2}(x) \phi_{1}^{\prime \prime}(x) \phi_{1}(x) d x \\
\alpha_{2}=\int_{0}^{l_{b}}\left[\phi_{1}^{\prime}(x) \psi_{1}^{\prime \prime}(x)+\phi_{1}^{\prime \prime}(x) \psi_{1}^{\prime}(x)\right] \phi_{1}(x) d x . \\
\alpha_{3}=\int_{0}^{l_{b}} \phi_{1}^{\prime}(x) \phi_{1}^{\prime \prime}(x) \psi_{1}(x) d x
\end{array}\right.
$$

For moving load and moving mass excitations, $f(t)=-M g \phi_{1}(v t)$, where, $M$ is the mass weight, $v$ is the speed of the moving force and $g$ denotes the gravitational acceleration. In the case of moving mass excitation, the governing equations of motions are as follows:

$$
\left\{\begin{array}{c}
\bar{M}(t) \ddot{A}(t)+\bar{C}(t) \dot{A}(t)+\bar{K}(t) A(t)- \\
E_{b} A_{b}\left(\frac{3}{2} \alpha_{1} A^{3}(t)+\alpha_{2} A(t) B(t)\right)=M g \phi_{1}(v t) . \\
\ddot{B}(t)+\bar{\omega}_{1}^{2} B(t)-E_{b} A_{b} \alpha_{3} A^{2}(t)=0
\end{array}\right.
$$

where,

$$
\left\{\begin{array}{l}
\bar{M}(t)=1+M \phi_{1}^{2}(v t) \\
\bar{C}(t)=2 v M \phi_{1}(v t) \phi_{1}^{\prime}(v t) \\
\bar{K}(t)=\omega_{1}^{2}+v^{2} M \phi_{1}(v t) \phi_{1}^{\prime \prime}(v t)
\end{array} .\right.
$$

For the moving load case the term, $M$, would vanish.

The same manipulation could be achieved to obtain the governing ordinary equations of motions for the controlled (smart) beam. To this end, by replacing Eq. 16 in Eq. 13 and following the same process, it yields to: 


$$
\left\{\begin{array}{l}
\ddot{A}(t)+\omega_{1}^{2} A(t)-E_{b} A_{b}\left(\frac{3}{2} \alpha_{1} A^{3}(t)+\alpha_{2} A(t) B(t)\right) \\
=f(t)+\frac{1}{2} \sum_{i=1}^{n}\left\{d_{31} E_{p}^{E} V_{i}(t) b_{p}\left(\begin{array}{l}
\left.\left[\begin{array}{ll}
\int_{L_{i-1}} \phi_{1}^{\prime \prime}(x) d x+\phi_{1}^{\prime}(x) \\
l_{L_{i-1}}^{h_{i}}
\end{array}\right]\right) \\
+\left.\left(h_{b}+h_{p}\right) \phi_{1}^{\prime}(x)\right|_{h_{2 i-1}} ^{h_{i}}
\end{array}\right]\right\} \\
\ddot{B}(t)+\bar{\omega}_{1}^{2} B(t)-E_{b} A_{b} A^{2}(t)=-\sum_{i=1}^{n}\left(\left.d_{31} E_{p}^{E} V_{i}(t) \psi_{1}(x)\right|_{L_{i-1}} ^{L_{i}}\right)
\end{array}\right.
$$

Herein, it is assumed the end condition of the beam is simple. Therefore, $\phi_{i}(x)=\sqrt{\frac{2}{\rho_{b} A_{b} l_{b}}} \sin \left(\frac{i \pi x}{l_{b}}\right), \omega_{i}=\left(\frac{i \pi}{l_{b}}\right)^{2} \sqrt{\frac{E_{b} I_{b}}{\rho_{b} A_{b}}}$, $i=1,2, \ldots, n_{1}$. The axial vibration mode shapes for an immovable-immovable boundary condition is:

$$
\left\{\begin{array}{l}
\psi_{i}(x)=\sqrt{\frac{2}{\rho_{b} A_{b} l_{b}}} \sin \left(\frac{i \pi x}{l_{b}}\right) \\
\bar{\omega}_{i}=\left(\frac{i \pi}{l_{b}}\right) \sqrt{\frac{E_{b}}{\rho_{b}}}, i=1,2, \ldots, n_{1} .
\end{array}\right.
$$

And for immovable-movable condition:

$$
\left\{\begin{array}{l}
\psi_{i}(x)=\sqrt{\frac{2}{\rho_{b} A_{b} l_{b}}} \sin \left(\frac{2 i-1}{2} \frac{\pi x}{l_{b}}\right) \\
\bar{\omega}_{i}=\frac{\pi}{2 l_{b}}(2 i-1) \sqrt{\frac{E_{b}}{\rho_{b}}}
\end{array}, i=1,2, \ldots, n_{1} .\right.
$$

By substituting Eqs. 25 and 26 in Eq. 21, the coefficients of $\alpha_{1}, \alpha_{2}$ and $\alpha_{3}$ are determined as shown in Table 1 .

In this study, ode45 solver of MATLAB software is employed to solve all of the obtained governing ordinary differential equations.

\section{Equivalent Linear Control Algorithm}

As it was mentioned earlier, there is no control algorithm developed for beam problems including geo- metrical nonlinear behavior. In this regard, by defining the non-dimensional displacement, velocity and acceleration parameters as:

$$
\left\{\begin{array} { l } 
{ \gamma _ { 1 } = \frac { A _ { \text { nonlin } } } { A _ { \text { lin } } } } \\
{ \gamma _ { 2 } = \frac { \dot { A } _ { \text { nonlin } } } { \dot { A } _ { \text { lin } } } } \\
{ \gamma _ { 3 } = \frac { \ddot { A } _ { \text { nonlin } } } { \ddot { A } _ { \text { lin } } } }
\end{array} \Rightarrow \left\{\begin{array}{l}
A_{\text {nonlin }}=\gamma_{1} A_{\text {lin }} \\
\dot{A}_{\text {nonlin }}=\gamma_{2} \dot{A}_{\text {lin }} . \\
\ddot{A}_{\text {nonlin }}=\gamma_{3} \ddot{A}_{\text {lin }}
\end{array}\right.\right.
$$

in which, the subscripts nonlin and lin signifies the parameters pertinent to the linear and nonlinear behaviors, correspondingly. The coefficients of $\gamma_{1}, \gamma_{2}$ and $\gamma_{3}$ are calculated online for each time instant. By replacing Eq. 27 into the motion equations of the controlled beam in the modal space, the Riccati-type equation (28) would be obtained as:

$$
\mathrm{P}_{e q} \overline{\mathrm{A}}_{e q}-\frac{1}{2} \mathrm{P}_{e q} \overline{\mathrm{D}} \mathrm{R}^{-1} \overline{\mathrm{D}}^{T} \mathrm{P}_{e q}+\overline{\mathrm{A}}_{e q}{ }^{T} \mathrm{P}_{e q}+2 \mathrm{Q}=0 .
$$

in the above equation, $\mathrm{R}$ and $\mathrm{Q}$ are the control weighting matrices which are semi-positive and positive definite ones, respectively. Besides, the subscript eq denotes the nonlinear parameter in which has been replaced by an equivalent linear one. For example, in the case of a moving mass excitation and assuming the first mode of vibration:

$$
\overline{\mathrm{A}}_{e q}=\left[\begin{array}{cc}
0 & \mathrm{I} \\
-\overline{\mathrm{M}}_{e q}^{-1} \overline{\mathrm{K}}_{e q} & -\overline{\mathrm{M}}_{e q}^{-1} \overline{\mathrm{C}}_{e q}
\end{array}\right],
$$

where,

$$
\left\{\begin{array}{l}
\bar{M}_{e q}=\gamma_{3}\left(1+M \phi_{1}^{2}(v t)\right) \\
\bar{C}_{e q}=2 v M \gamma_{2} \phi_{1}(v t) \phi_{1}^{\prime}(v t) . \\
\bar{K}_{e q}=v^{2} M \gamma_{1} \phi_{1}(v t) \phi_{1}^{\prime \prime}(v t)
\end{array}\right.
$$

After determination of $\mathrm{P}_{e q}$, at any time instant, the control gain matrix of the system could be found as:

$$
\mathrm{G}_{e q}=-\frac{1}{2} \mathrm{R}^{-1} \overline{\mathrm{D}}^{T} \mathrm{P}_{e q}
$$

Table 1. The constant coefficients of $\alpha_{1}, \alpha_{2}$ and $\alpha_{3}\left(a=\sqrt{\frac{2}{\rho_{b} A_{b} l_{b}}}\right)$.

\begin{tabular}{lccc}
\hline Beam's axial end condition & $\alpha_{1}$ & $\alpha_{2}$ & $\alpha_{3}$ \\
\hline Immovable-immovable & $-\frac{a^{4} l_{b}}{8}\left(\frac{\pi}{l_{b}}\right)^{4}$ & 0 & 0 \\
Immovable- movable & $-\frac{a^{4} l_{b}}{8}\left(\frac{\pi}{l_{b}}\right)^{4}$ & $-0.1485446 a^{3} l_{b}\left(\frac{\pi}{l_{b}}\right)^{3}$ & $0.08488264 a^{3} l_{b}\left(\frac{\pi}{l_{b}}\right)^{3}$ \\
\hline
\end{tabular}


Finally, the required control voltages in the piezoelectric patches are calculated as:

$$
\mathrm{u}(t)=\mathrm{G}_{e q} \mathrm{X}(t) .
$$

in which, $\mathrm{u}(t)$ contains the required control voltages and, $X(t)=\left[\begin{array}{c}A_{\text {nonlin }} \\ \dot{A}_{\text {nonlin }}\end{array}\right]$.

\section{Numerical Example}

A simply supported uniform prismatic aluminum beam is assumed, in which, $E_{b}=6.5 \times 10^{10} \mathrm{pa}, \rho_{b}=2700 \mathrm{kgm}^{-3}$, $h_{b}=0.05 \mathrm{~m}$ and $b_{b}=0.10 \mathrm{~m}$. As it is shown in Figure 2, a single piezoelectric patch is bonded on the lower surface of the beam mid-point. This patch is assumed to be a PZT-5H, where in, $E_{p}^{E}=6.2 \times 10^{10} \mathrm{~Pa}$, $\rho_{p}=7800 \mathrm{kgm}^{-3}, d_{31}=-320 \times 10^{-12} \mathrm{~m} / \mathrm{V}$. Three different lengths for the piezoelectric actuator are supposed i.e., $l_{p}=0.05 l_{b}, 0.10 l_{b}, 0.15 l_{b}$, and also, $h_{p}=0.005 \mathrm{~m}$ and $b_{p}=b_{b}=0.10 \mathrm{~m}$. As it was noted in section 4, two axial boundary conditions, i.e., Immovable-Immovable (Figure 2a) and Immovable-Movable (Figure 2b) is considered in all nonlinear analyses.

The external excitations are assumed to be moving load and moving mass. Therefore, in Figure 3, the dynamic amplification factor of the beam mid-point deflection is depicted as a function of moving load weight and velocity for both linear and nonlinear analyses considering the first mode of vibration. According to this figure, the obtained results pertinent to the nonlinear analysis stand lower than those obtained for linear analysis for both types of loadings. This is because of the added nonlinear stiffness. On the other hand, with an increase on weight and velocity of the moving mass, the difference between two analyses becomes clearer, especially for the moving mass case. However, the linear results make an upper bound for

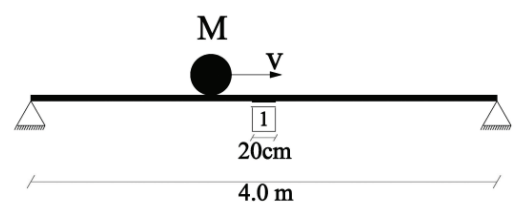

(a)

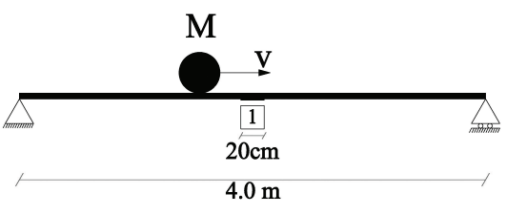

(b)

Figure 2. Single span smart beam with a single piezoelectric actuator at its mid-point; (a) axially immovable-immovable, (b) axially immovable-movable.
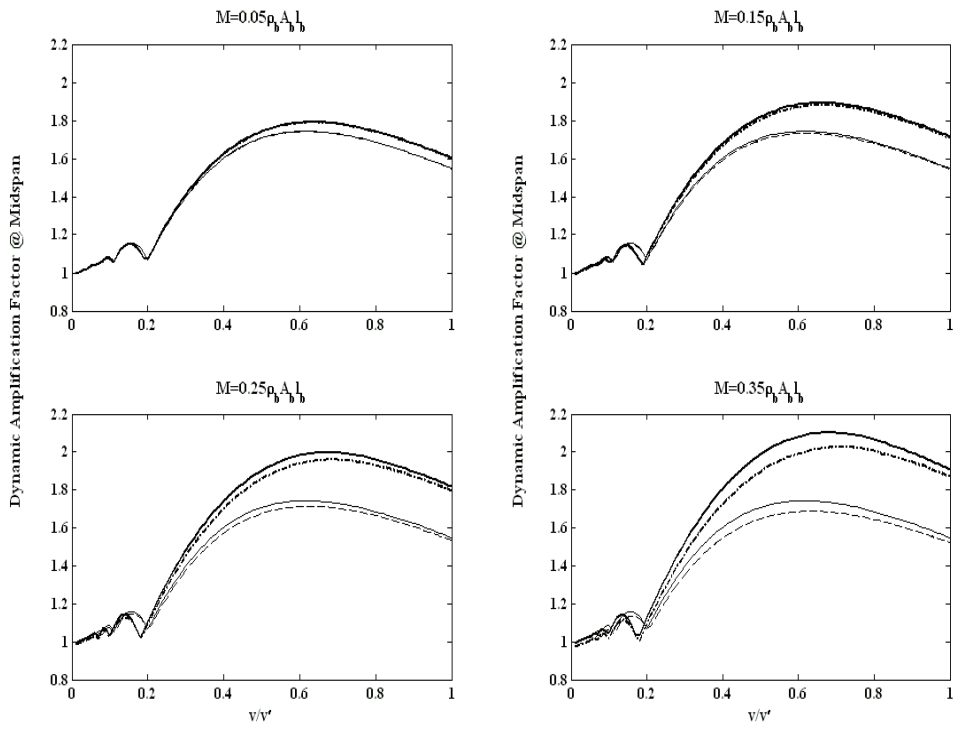

Figure 3. Variation of the dynamic amplification factor as a function of moving mass velocity ( — linear analysis; nonlinear analysis), immovable-immovable, -..-. nonlinear analysis, immovable-movable (bold lines signifies moving mass excitation and the pale lines signify the moving load). 
the nonlinear ones, for most of the considered intervals. Furthermore, the axial boundary conditions seem to have no appreciable effects.

In Figures 4-6, the normalized linear and nonlinear deflections, velocities and accelerations time histories of the beam mid-span are depicted for $M=0.25 \rho_{b} A_{b} l_{b}$ and $v=0.20 v^{\prime}, 0.50 v^{\prime}, 0.8 v^{\prime}$. To this end, the deflection parameter is normalized by the static deflection defined as, $\Delta_{\text {static }}=\frac{M g l_{b}^{3}}{E_{b} I_{b}}$ while the velocity and acceleration parameters are normalized by $\Delta_{\text {static }} / T_{1}$ and $\Delta_{\text {static }} / T_{1}^{2}$, respectively, wherein, $T_{1}=2 \pi\left(\frac{\pi}{l_{b}}\right)^{2} \sqrt{\frac{\rho_{b} A_{b} l_{b}}{E_{b} I_{b}}}$. Expectedly, the difference between two analyses is more distinct as the moving load velocity increases. Besides, the phase difference of two analyses is more evident for higher velocities of the force.

To control the response of the beam, the control target is assumed to be a $25 \%$ decrease in the beam dynamic deflection at its mid-span. In this regard, the required control voltage for the piezoelectric actuator of the length, $0.05 l_{b}$, is calculated for both, linear and nonlinear behaviors of the host structure. In Figure 7, the uncontrolled as well as controlled deflection of the beam is illustrated. As it could be observed, the performance of the proposed control algorithm is similar to that utilized for the linear behavior of the beam, in which, both of them causes the
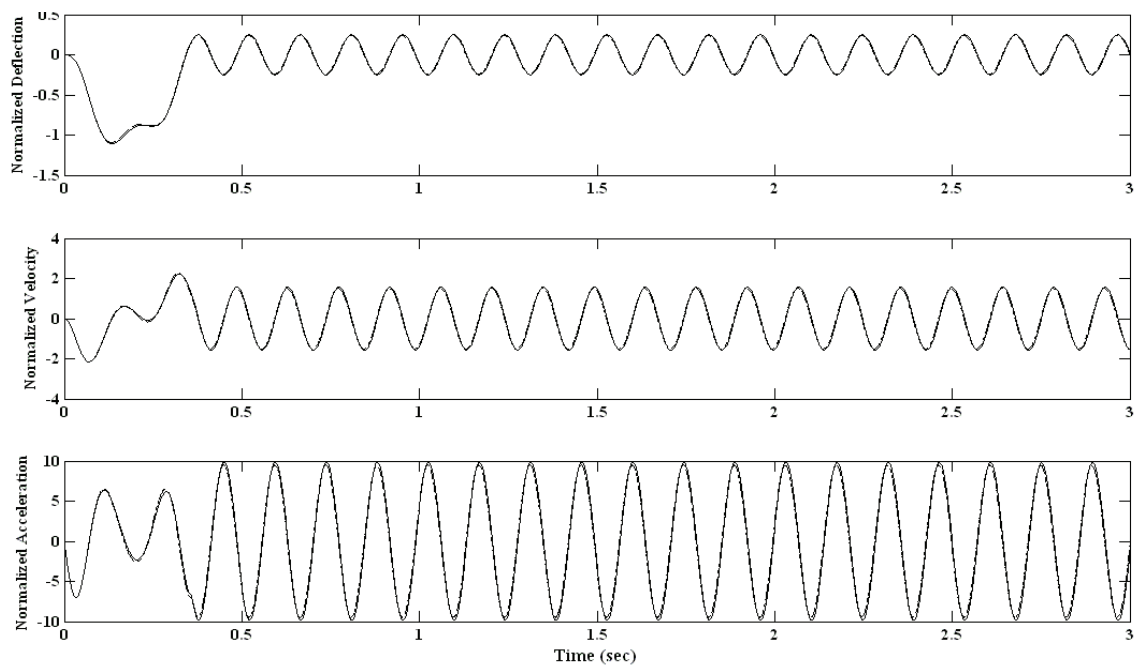

Figure 4. Variation of the normalized kinematic parameters of the structural system vs. time at the beam mid-span for the moving mass excitation, $M=0.25 \rho_{b} A_{b} l_{b}, v=0.20 v^{\prime}$ ( — linear analysis; .......... nonlinear analysis, immovable-immovable, -..-. nonlinear analysis, immovable-movable).
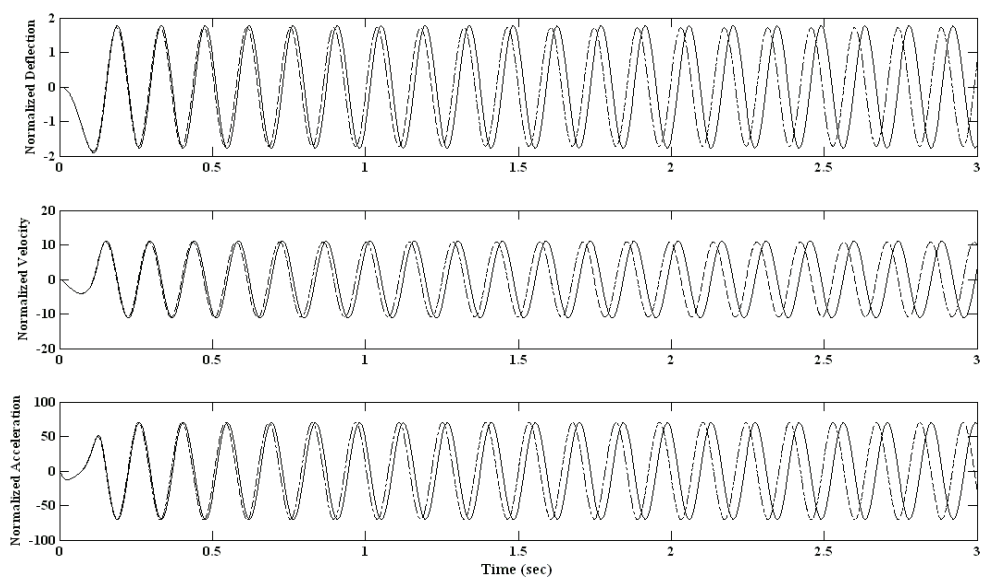

Figure 5. Variation of the normalized kinematic parameters of the structural system vs. time at the beam mid-span for the moving mass excitation, $M=0.25 \rho_{b} A_{b} l_{b}, v=0.50 v^{\prime}$ ( — linear analysis, .......... nonlinear analysis, immovable-immovable, -.-.. nonlinear analysis, immovable-movable). 

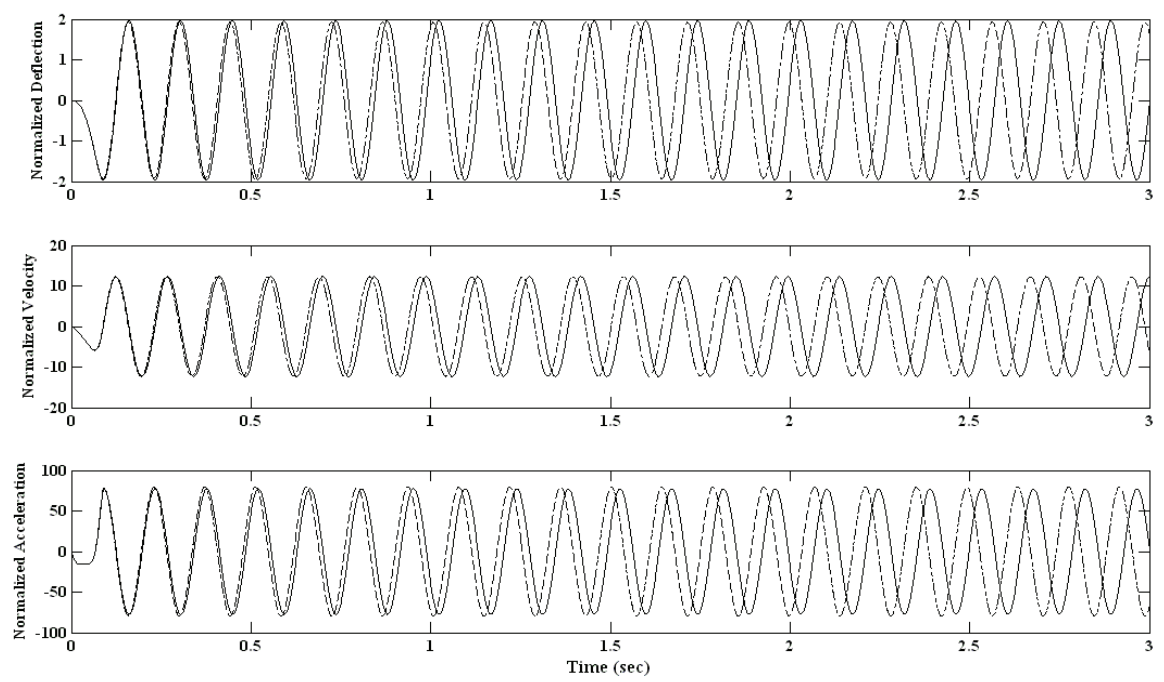

Figure 6. Variation of the normalized kinematic parameters of the structural system vs. time at the beam mid-span for the moving mass excitation, $M=0.25 \rho_{b} A_{b} l_{b}, v=0.80 v^{\prime}$ ( — linear analysis, ......... nonlinear analysis, immovable-immovable, -...-. nonlinear analysis, immovable-movable).
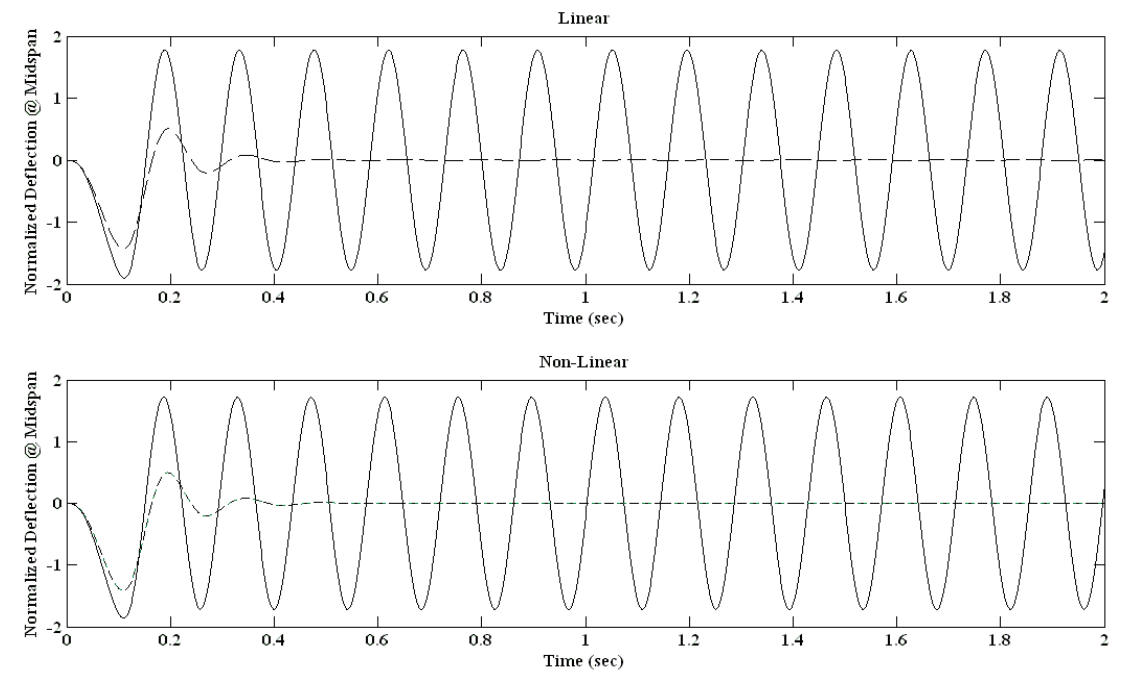

Figure 7. Deflection time history of the uncontrolled and controlled beams mid-point under a moving mass, $M=0.25 \rho_{b} A_{b} l_{b}$ , $v=0.50 v^{\prime}$ ( - uncontrolled, ------ controlled-linear, Uncontrolled-nonlinear, immovable-immovable, -.-.. controlled-nonlinear, immovable-movable).

response of the structure suppresses quickly. Achieving this control target, $\mathrm{R}=1.0 \times 10^{-4} \mathrm{I}_{1 \times 1}$ and $\mathrm{Q}=3.5 \times 10^{4} \mathrm{I}_{2 \times 2}$ for linear and nonlinear behaviors. In Figure 8, the variation of the required control voltage in the piezoelectric actuator is shown. As it is obvious, the required control voltage for both analyses are quiet coincident and therefore, if the beam is controlled based on the linear behavior, it would be completely trustable for nonlinear one, either.

Investigating the effect of the piezoelectric patch length on the required control voltage is subject of the
Figure 9. In this regard, by maintaining the same control target, the length of the actuator is assumed to be $0.10 l_{b}$ and $0.20 l_{b}$. The control weight matrices are defined as $\mathrm{R}=2.0 \times 10^{-4} \mathrm{I}_{1 \times 1}, \quad \mathrm{Q}=1.75 \times 10^{4} \mathrm{I}_{2 \times 2}$ for $l_{p}=0.10 l_{b}$ and $\mathrm{R}=4.0 \times 10^{-4} \mathrm{I}_{1 \times 1}, \mathrm{Q}=9 \times 10^{3} \mathrm{I}_{2 \times 2}$ for $l_{p}=0.20 l_{b}$, as well. According to this figure, the maximum required control voltage is in a fairly converse linear relation with the length of the actuator. However, the increase of the patch length should be limited as the location of the neutral axis of the beam is to be unchanged except for a small length of the beam. 


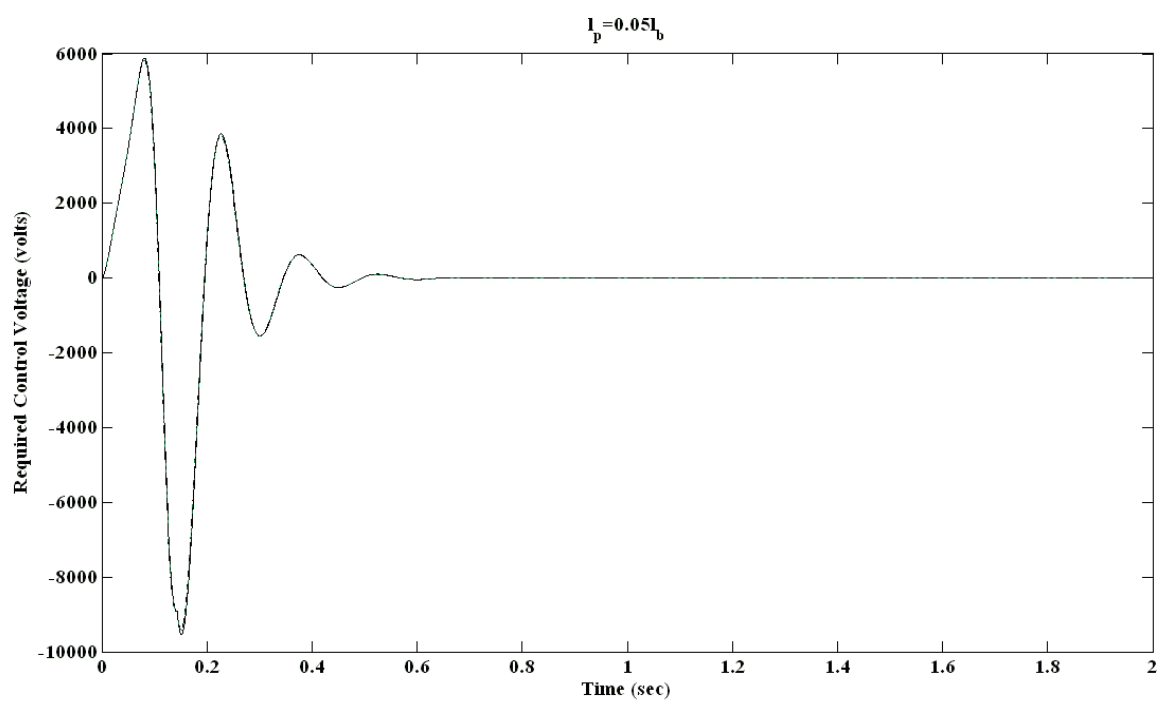

Figure 8. Varitation of the required voltage in the piezoelectric actuator with a length of $0.05 l_{b}$ for a moving mass excitation, $M=0.25 \rho_{b} A_{b} l_{b}, v=0.50 v^{\prime}$ ( immovable-movable).
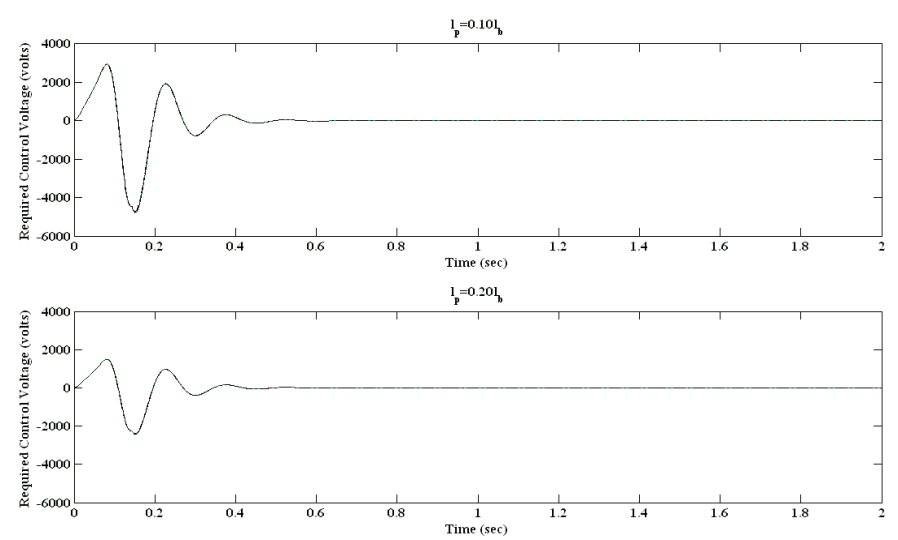

Figure 9. Variation of the required voltage in the piezoelectric actuators with the lenghts of $0.10 l_{b}$ and $0.20 l_{b}$ for a moving mass excitation, $M=0.25 \rho_{b} A_{b} l_{b}, v=0.50 v^{\prime}$ ( analysis, immovable-movable).

\section{Conclusion}

The governing differential equations of motions of an Euler-Bernoulli beam with a number of piezoelectric patches bonded on its lower side with geometrically nonlinear behavior was derived based on the Hamilton's principle. Employing the Eigen function expansion method with consideration of the first vibration mode, an equivalent linear control algorithm was proposed. The axial boundary conditions were assumed to be immovable-immovable and immovable-movable and the beam for the numerical examples was supposed to be simply supported. The obtained results of the uncontrolled beam under a moving load and moving mass excitations showed the response pertaining to the nonlinear analysis is smaller than the linear one according to the augmented nonlinear stiffness. Utilizing a single piezoelectric actuator at the beam mid-span, the deflection of the beam was decreased into some required levels via linear and equivalent linear control algorithms. It was proved the proposed algorithm is efficient; however, the linear algorithm could be used as a trustable and stable algorithm for nonlinear behavior of the host beam. The axial boundary conditions effect on the results was shown to be negligible. Finally, by increasing the length of the actuator, the maximum required control voltage reduced. However, the length of 
the actuator is restricted because of the basic assumption to maintain the location of the neutral line of the beam.

\section{Acknowledgement}

Special thanks are given to the Research Center of Islamic Azad University, Chalous branch, for financial supports in this study.

\section{References}

1. Fryba L. Vibration of solids and structures under moving loads. 3rd ed. London: Thomas Telford; 1999.

2. Esmailzadeh E, Ghorashi M. Vibration analysis of beams traversed by uniform partially distributed moving masses. J Sound Vib. 1995; 184(1):9-17.

3. Ichikawa M, Miyakawa Y, Matsuda A. Vibration analysis of the continuous beam subjected to a moving mass. J Sound Vib. 2000; 230(3):493-506.

4. Bilello C, Bergman LA, Kuchma D. Experimental investigation of a small-scale bridge model under a moving mass. J Struct Eng, ASCE. 2004; 130(5):799-804.

5. Nikkhoo A, Rofooei FR, Shadnam MR. Dynamic behavior and modal control of beams under moving mass. J Sound Vib. 2007; 306(3-5):712-24.

6. Kiani K, Nikkhoo A, Mehri B. Prediction capabilities of classical and shear deformable beam theories excited by a moving mass. J Sound Vib. 2009; 320(3):632-48.

7. Kiani K, Nikkhoo A, Mehri B. Parametric analyses of multispan viscoelastic shear deformable beams under excitation of a moving mass. J Vib Acoust, ASME. 2009; 131(5):051009.

8. Kiani K, Nikkhoo A, Mehri B. Assessing dynamic response of multispan viscoelastic thin beams under a moving mass via generalized moving least square method. Acta Mech Sin. 2010; 26(5):721-33.

9. Zarfam R, Khaloo AR, Nikkhoo A. On the response spectrum of Euler-Bernoulli beams with a moving mass and horizontal support excitation. Mech Res Comm. 2013; 47:77-83.

10. Eftekhar Azam S, Mofid M, Afghani Khoraskani R. Dynamic response of Timoshenko beam under moving mass. Scientia Iranica. 2013; 20(1):50-56.

11. Ebrahimzadeh Hassanabadi M, Nikkhoo A, Vaseghi Amiri J, Mehri B. A new orthonormal polynomial series expansion method in vibration analysis of thin beams with non-uniform thickness. Appl Math Model. 2013; 37 (18-19):8543-56.

12. Ahmadi M, Nikkhoo A. Utilization of characteristic polynomials in vibration analysis of non-uniform beams under a moving mass excitation. [In Press]. Appl Math Model. 2013 Oct. Available from: http://dx.doi.org/10.1016/j. apm.2013.10.011
13. Gbadeyan JA, Oni ST. Dynamic behavior of beams and rectangular plates under moving loads. J Sound Vib. 1995; 182(5):677-95.

14. Shadnam MR, Mofid M, Akin JE. On the dynamic response of rectangular plate, with moving mass. Thin Wall Struct. 2001; 39(9):797-806.

15. Nikkhoo A, Rofooei FR. Parametric study of the vibration of thin rectangular plates traversed by a moving mass. Acta Mech. 2012; 223(1):15-27.

16. Amiri JV, Nikkhoo A, Davoodi MR, Ebrahimzadeh Hassanabadi M. Vibration analysis of a mindlin elastic plate under a moving mass excitation by eigen function expansion method. Thin Wall Struct. 2013 Jan; 62:53-64.

17. Nikkhoo A, Ebrahimzadeh Hassanabadi M, Eftekhar Azam S, Vaseghi Amiri J. Vibration of a thin rectangular plate subjected to series moving inertial loads. Mech Res Comm. 2014 Jan; 55:105-1123. Available from: http://dx.doi. org/10.1016/j.mechrescom.2013.10.009.

18. Soong TT. State-of-the-art-review: active structural control in civil engineering. Eng Struct. 1988; 10(90):74-84.

19. Song G, Sethi V, Li HN. Vibration control of civil structures using piezoceramic smart materials: a review paper. Eng Struct. 2006; 28(11):1513-24.

20. Sung YG. Modeling and control with piezoactuators for a simply supported beam under a moving mass. J Sound Vib. 2002; 250(4):617-626.

21. Rofooei FR, Nikkhoo A. Application of active piezoelectric patches in controlling the dynamic response of a thin rectangular plate under moving mass. Int J Solid Struct. 2009; 46(11-12):2429-43.

22. Nikkhoo A. Investigating the behavior of smart thin beams with piezoelectric actuators under dynamic loads. Mech Syst Signal Process. 2014 Apr; 45(2):513-30. Available from: http://dx.doi.org/10.1016/j.ymssp.2013.11.003.

23. Hino J, Yoshimura T, Ananthanarayana N. Vibration analysis of non-linear beams subjected to a moving load using the finite element method. J Sound Vib. 1985; 100(4):477-91.

24. Yoshimura T, Hino J, Ananthanarayana N. Vibration analysisofanon-linearbeamsubjected tomovingloadsbyusingthe Galerkin method. J Sound Vib. 1986; 104(2):179-86.

25. Kiani K, Nikkhoo A. On the limitations of linear thin beam theory for the problem of moving mass-beam interaction. Acta Mech Sin. 2012; 28(1):1-16.

26. IEEE. 176-1987 - IEEE Standard on Piezoelectricity. New York: Institute of Electrical and Electronics Engineers Inc; 1988.

27. Park CH. Dynamic modeling of beams with shunted piezoelectric elements. J Sound Vib. 2003; 268(1):115-29.

28. Nayfeh AH, Mook DT. Nonlinear oscillations. New York: John Wiley \& Sons; 1979.

29. Crawely EF, de Luis J. Use of piezoelectric actuators as elements of intelligent structures. AIAA Journal. 1987; 25(10):1373-85. 